Cumhuriyet Üniversitesi Fen Fakültesi Fen Bilimleri Dergisi (CFD), Cilt 37, No. 2 (2016) ISSN: $1300-1949$
Cumhuriyet University Faculty of Science

Science Journal (CSJ), Vol. 37, No. 2 (2016) ISSN: $1300-1949$

http://dx.doi.org/10.17776/csj.37809

\title{
Parabolik Kuantum Kuyusundaki Hidrojenik Düzeyler Üzerine Manyetik Alan Etkisi
}

\author{
Ünal. YEŞİLGÜL ${ }^{1 *}$, Cihat.ÇOBAN ${ }^{2}$, Esin KASAPOĞLU² \\ ${ }^{I}$ Teknoloji Fakültesi, Optik Mühendisliği Bölümü, Cumhuriyet Üniversitesi, 58140 Sivas, Türkiye \\ ${ }^{2}$ Fen Fakültesi, Fizik Bölümü, Cumhuriyet Üniversitesi, 58140 Sivas, Türkiye
}

Received: 19.01.2016; Accepted: 05.03.2016

\begin{abstract}
Özet. Bu çalışmada, tek parabolik kuantum kuyusunda $1 \mathrm{~s}, 2 \mathrm{~s}$ ve $2 \mathrm{p} \pm$ düzeylerindeki donor safsızlık atomunun bağlanma enerjisi, etkin kütle yaklaşımında varyasyonel olarak, kuyu genişliği ve dışarıdan uygulanan manyetik alanın bir fonksiyonu olarak hesaplamıştır. Ayrıca, GaAs/Ga1-xAlxAs tek parabolik kuantum kuyusunda hidrojenik düzeyler arasındaki kızılötesi geçişler üzerinde manyetik alanın etkisi incelenmiştir.
\end{abstract}

Anahtar Kelimeler: Parabolik kuantum kuyusu, Safsızlık bağlanma enerjisi, Manyetik alan

\section{Effect of Magnetic Field on Hydrogenic Energy Levels in Parabolic Quantum Well}

\begin{abstract}
In this study, the binding energy of the donor impurity at $1 \mathrm{~s}, 2 \mathrm{~s}$ and $2 \mathrm{p} \pm$ states in a parabolic quantum well is calculated variationally within the effective mass approximation as a function of the quantum well width and the external magnetic field. Furthermore, the effects of the magnetic field on the infrared transitions between the hydrogenic states in a parabolic quantum well under are investigated.
\end{abstract}

Keywords: Parabolic Quantum Well, Impurity Atoms, Magnetic Fields

\section{GİRIŞ̧}

Yarıiletkenlerin kristal yapılarının incelenmesi sayesinde yarilletkenlerin optik ve elektronik özellikleri belirlenebilmektedir. Moleküler kimyasal buhar depolama (MOCVD), moleküler ışın epitaksi (MBE), buhar faz epitaksi (VPE), sıvı faz epitaksi (LPE) gibi epitaksiyel büyütme teknikleri yariiletken fiziğine önemli etkiler yapmıştır. Bu epitaksiyel büyütme teknolojisindeki gelişmeler, parabolik, yarı-parabolik, V-şekilli kuşatma potansiyelleri gibi farklı şekillerde yariiletken kuantum kuyuları üretme imkânı vermiştir. Bu sistemlerde, yapının optik ve elektronik özellikleri külçe yapılarına göre oldukça değişmektedir. Bu düşük boyutlu yarıiletken yapılar elektronik ve optoelektronik aygitlardaki potansiyel uygulamaları ve benzersiz fiziksel özellikleri nedeni ile yoğun bir şekilde çalışılmaktadır[1-10].

Yarıiletken heteroyapıların elektronik ve optik özellikleri üzerinde donor safsızlık atomlarının (impuritilerin) etkisinin araştırılması yarıiletken fiziğinin önemli bir konusudur. Safsızlık atomlarının varlığı kuantum cihazlarının performansını belirgin şekilde değiştirir. Bu nedenle birçok bilim insanı bu konu üzerinde yoğun bir şekilde çalışmıştır[11-14]. Bununla birlikte; manyetik alan, elektrik alan ve yoğun lazer alanı gibi dışarıdan uygulanan dış etkilerin sistemin elektronik ve optik özellikleri üzerinde

\footnotetext{
* Corresponding author. Email address: uyesilgul@ cumhuriyet.edu.tr 
önemli değişmelere yol açtığı gözlenmiştir. Manyetik alanın uygulanması safsızlık durumlarının simetrisini ve dalga fonksiyonunun doğasını değiştirdiği için bağlanma enerjisinde çok belirgin değişiklikler ortaya çıkarır. Bu nedenle farklı potansiyel geometrilerine sahip kuantum kuyularında kuşatılan hidrojenik donorlar üzerinde manyetik alan etkileri yoğun olarak araştırılmaktadır [15- 20].

$\mathrm{Bu}$ çalışmada, büyütme doğrultusuna paralel olarak uygulanan manyetik alan altındaki $G a_{1-x} A l_{x} A s / G a A s$ parabolik kuantum kuyusundaki safsızlık atomlarının bağlanma enerjileri ve hidrojenik düzeyler arasındaki geçiş enerjileri etkin kütle yaklaşımı çerçevesinde varyasyonel yöntem kullanılarak incelendi.

\section{TEORI}

Üzerinde çalışlan sistem şemetik gösterimi Şekil 1'de verilen GaAs-tabanlı, z-doğrultusu boyunca büyütülen parabol biçimli tek kuantum kuyusundan oluşur. Etkin kütle yaklaşımı çerçevesinde, +zyönünde uygulanan manyetik alan altındaki elektron-safsızlık sisteminin Hamiltonian'i Denklem 1' deki gibidir

$$
H=\frac{\left(\vec{p}+\frac{e \vec{A}}{c}\right)^{2}}{2 m^{*}}+V(z)-\frac{e^{2}}{\varepsilon_{0} \sqrt{\left(x-x_{i}\right)^{2}+\left(y-y_{i}\right)^{2}+\left(z-z_{i}\right)^{2}}}
$$

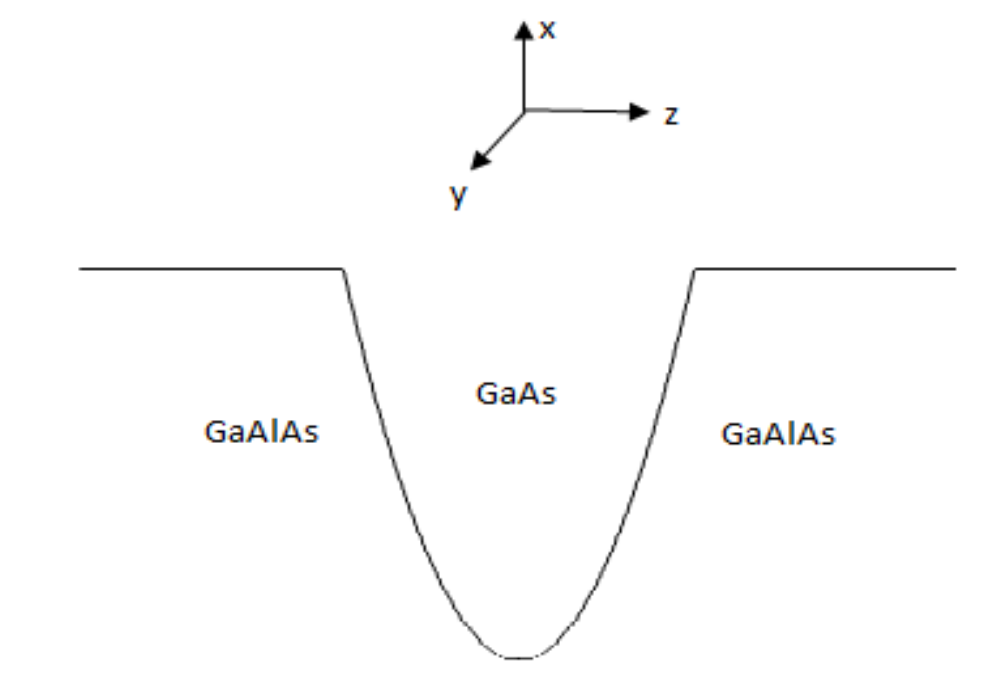

Şekil 1. Parabolik kuantum kuyusunun şematik gösterimi.

Burada $\vec{p}$ elektronun momentumunu, $e$ elektronun yükünü, $\varepsilon_{\mathrm{o}}$ ortamın dielektrik katsayısı, $\left(x_{i}, y_{i}, z_{i}\right)$ safsızlık atomunun konumu ve (x, y, z) elektronun konumunu göstermektedir. 
$\vec{A}=\left(-\frac{B_{0} y}{2}, \frac{B_{0} x}{2}, 0\right)$ biçiminde tanımlı vektör potansiyeli, $V(z)$ kuşatma potansiyelidir ve potansiyelin fonksiyonel ifadesi aşağıdaki gibidir.

$$
V(z)=\left\{\begin{array}{cl}
\frac{4 V_{0} z^{2}}{L^{2}} ; & |z| \leq \frac{L}{2} \\
V_{0} ; & |z|>\frac{L}{2}
\end{array}\right.
$$

$\vec{p}$ operatörünün ve $\vec{A}$ vektörünün potansiyel değerleri Denklem 1'de yazılırsa sistemin Hamiltonian

$$
\begin{array}{r}
H=-\frac{\hbar^{2}}{2 m^{*}}\left[\frac{d^{2}}{d x^{2}}+\frac{d^{2}}{d y^{2}}+\frac{d^{2}}{d z^{2}}\right]-\frac{e \hbar B_{0} L_{z}}{2 m^{*} c}+\frac{e^{2} B_{0}^{2}\left(x^{2}+y^{2}\right)}{8 m^{*} c^{2}} \\
-\frac{e^{2}}{\varepsilon_{0} \sqrt{\left(x-x_{i}\right)^{2}+\left(y-y_{i}\right)^{2}+\left(z-z_{i}\right)^{2}}}+V(z)
\end{array}
$$

şeklinde elde edilir. Burada $L_{z}$, açısal momentum operatörünün $\hbar=1$ birimdeki z-bileşenidir.

$x=\rho \cos \phi$

$y=\rho \sin \phi$

$z=z$

dönüşümü yapılarak kutupsal koordinatlardaki Hamiltonian ifadesi;

$$
\begin{aligned}
H=\frac{\hbar^{2}}{2 m^{*}}\left[\frac{d^{2}}{d \rho^{2}}\right. & \left.+\frac{1}{\rho^{2}} \frac{d^{2}}{d \phi^{2}}+\frac{1}{\rho} \frac{d}{d \rho}+\frac{d^{2}}{d z^{2}}\right]-\frac{e B_{0} L_{z}}{2 m^{*} c}+\frac{e^{2} B_{0}^{2} \rho^{2}}{8 m^{*} c^{2}}-\frac{e^{2}}{\varepsilon_{0} \sqrt{\rho^{2}+\left(z-z_{i}\right)^{2}}} \\
& +V(z)
\end{aligned}
$$

olarak elde edilir. Burada $\rho=\sqrt{\left(x-x_{i}\right)^{2}+\left(y-y_{i}\right)^{2}}$, (x-y) düzlemindeki elektron-safsızlık arasındaki bağıl uzaklıktır. Hamiltonian'deki tüm uzunluklar Bohr yarıçapı $\left(a_{B}=\frac{\varepsilon_{0} \hbar^{2}}{m^{*} e^{2}}\right)$ ve tüm enerji terimleri Rydberg $\left(R=\frac{m^{*} e^{4}}{2 \varepsilon_{0}^{2} \mathrm{~h}^{2}}\right)$ cinsinden yazılarak Hamiltonian boyutsuz hale getirilir. Boyutsuz Hamiltonian;

$$
\widetilde{H}=-\left[\frac{d^{2}}{d \tilde{\rho}^{2}}+\frac{1}{\tilde{\rho}^{2}} \frac{d^{2}}{d \phi^{2}}+\frac{1}{\tilde{\rho}} \frac{d}{d \tilde{\rho}}\right]-\tilde{Y} L_{z}+\frac{1}{4} \tilde{\gamma}^{2} \tilde{\rho}^{2}-\frac{2}{\sqrt{\tilde{\rho}^{2}+\left(\tilde{z}-\tilde{z}_{i}\right)^{2}}}
$$

biçiminde yazılır. Burada $\tilde{Y}=\frac{e \hbar^{2} B_{0}}{2 m c R}$ ile verilir ve birinci Landau düzeyi olarak adlandırılır. Sistemin Schrödinger denklemi;

$$
\widetilde{H} \psi(\tilde{z}, \tilde{\rho}, \alpha)=\tilde{E} \psi(\tilde{z}, \tilde{\rho}, \alpha)
$$


biçimindedir. Burada $\psi(\tilde{z}, \tilde{\rho}, \lambda)$ dalga fonksiyonu

$$
\psi(\tilde{z}, \tilde{\rho}, \lambda)=\varphi(\tilde{z}) \phi(\tilde{z}, \tilde{\rho}, \lambda)
$$

ile verilir. $\varphi(\tilde{z})$, z-doğrultusundaki Schrödinger denkleminin çözümünden elde edilen dalga fonksiyonu, $\phi(\tilde{z}, \tilde{\rho}, \lambda)$ ise; $1 \mathrm{~s}, 2 \mathrm{~s}$ ve $2 p_{ \pm}$düzeylerini temsil eden hidrojenik dalga fonksiyonudur ve ilgili düzeylere ait dalga fonksiyonları aşağıdaki gibidir.

$$
\begin{aligned}
& \phi_{1 s}\left(\tilde{z}, \tilde{\rho}, \lambda_{1}\right)=N_{1} e^{-\frac{\sqrt{\tilde{\rho}^{2}+\left(\tilde{z}-\tilde{z}_{i}\right)^{2}}}{\lambda_{1}}} \\
& \phi_{2 s}\left(\tilde{z}, \tilde{\rho}, \beta, \lambda_{2}\right)=N_{2}\left(1-\beta \sqrt{\tilde{\rho}^{2}+\left(\tilde{z}-\tilde{z}_{i}\right)^{2}}\right) e^{-\frac{\sqrt{\tilde{\rho}^{2}+\left(\tilde{z}-\tilde{z}_{i}\right)^{2}}}{\lambda_{2}}} \\
& \phi_{2 p_{ \pm}}\left(\tilde{z}, \tilde{\rho}, \phi, \lambda_{3}\right)=N_{3} \tilde{\rho} e^{-\frac{\sqrt{\tilde{\rho}^{2}+\left(\tilde{z}-\tilde{z}_{i}\right)^{2}}}{\lambda_{3}}}
\end{aligned}
$$

Burada $N_{1}, N_{2 \text { ve }} N_{3}$ normalizasyon katsayılar1, $\lambda_{1}, \lambda_{2}$, ve $\lambda_{3}$ varyasyon parametreleri ve $\beta, 1 \mathrm{~s}$ ve $2 \mathrm{~s}$ deneme dalga fonksiyonlarının ortogonallik koşulundan belirlenebilen bir sabittir.

$\mathrm{z}$ doğrultusundaki dalga fonksiyonu $\varphi(\tilde{z})$, genişliği $\mathrm{L}_{\mathrm{b}}=2 \mathrm{~L}$ olan sonsuz potansiyel kuyusunun öz fonksiyonlarından oluşan çözümler baz alınarak oluşturulmuştur. Sözü edilen bazlar genel formda,

$$
\varphi_{n}(\tilde{z})=\sqrt{\frac{2}{L_{b}}} \cos \left[\frac{n \pi}{L_{b}} \tilde{z}-\delta_{n}\right]
$$

biçiminde elde edilir. Burada $\delta_{n}, \mathrm{~L}_{\mathrm{b}}$ genişliğindeki sonsuz kuantum kuyusunun öz fonksiyonlarındaki tek ve çift özdeğerlere karşılık gelen faz farkı olmak üzere;

$$
\delta_{n}= \begin{cases}0 ; & n: \text { tek ise } \\ \frac{\pi}{2} ; & n: \text { çift ise }\end{cases}
$$

değerlerini alır. Bu durumda sistemi tanımlayan dalga fonksiyonu,

$$
\varphi(\tilde{z})=\sum_{n=0}^{\infty} C_{n} \varphi_{n}(\tilde{z})
$$

biçimindedir. Sistemin toplam enerjisi ise;

$$
\tilde{E}=\min _{\lambda}\langle\psi|\widetilde{H}| \psi\rangle
$$

varyasyon teoreminde elde edilir. Bu eşitlik sayesinde $\tilde{E}$ özdeğerini minimum yapan $\lambda$ varyasyon parametreleri belirlenir. $1 \mathrm{~s}(\mathrm{~m}=0), 2 \mathrm{~s}(\mathrm{~m}=0)$ ve $2 p_{ \pm}(\mathrm{m}= \pm 1)$ durumunda bulunan safsizlik atomunun bağlanma enerjisi aşağıdaki denklem şeklinde verilir[21]. 


$$
\tilde{E}_{B}=\widetilde{E}_{z}+(m+|m|+1) \tilde{Y}-<\widetilde{H}>
$$

\section{SONUÇLAR VE TARTIŞMA}

Hesaplamalarda kullanılan fiziksel parametrelerin değerleri $\mathrm{m}^{*}=0.0665 \mathrm{~m}_{0}\left(\mathrm{~m}_{\mathrm{o}}\right.$ serbest elektron kütlesi), ortamın dielektrik katsayısı olan $\varepsilon_{0}=12.58$, iletkenlik bandı süreksizliği $V_{o}=228 \mathrm{meV}$, safsızlık atomu konumu $\left(\mathrm{x}_{\mathrm{i}}=\mathrm{y}_{\mathrm{i}}=\mathrm{z}_{\mathrm{i}}=0\right)$ olarak alınmıştır.

Çalışmamız da ilk olarak kuyu genişliği L ve manyetik alan şiddeti değiştirilerek kuyu merkezinde yerleşen donor safsızlı atomu için $1 \mathrm{~s}, 2 \mathrm{~s}$ ve $2 \mathrm{p}_{ \pm}$düzeylerinin bağlanma enerjilerinin değişimi incelenmiş ve sonuçlar Şekil 2, Şekil 3 ve Şekil 4'de verilmiştir. Şekil 2 açıkça görüldüğü gibi kuyu genişliği arttıkça bağlanma enerjisi önce bir maksimuma ulaşıncaya kadar artar ve sonra azalmaya başlar. Bunun nedeni şu şekilde açıklayabiliriz; bağlanma enerjisinin maksimum olduğu L değerleri için elektron safsızlık atomunun aynı düzlemde bulunma olasılığı fazladır. Çok büyük L değerlerinde ise büyütme doğrultusundaki kuşatma azalır ve safsızlık atomunun bağlanma enerjisi üç boyutlu sistemdeki değerine yakınlaşır. Bu nedenle geniş kuyularda bağlanma enerjisi azalır. Ayrıca bu şekillerde açıkça görüldüğü gibi manyetik alanın artması ile bağlanma enerjisi artmaktadır. Manyetik alan (x-y) düzleminde bir kuşatma meydana getirir. Elektronun (x-y) düzleminde kuşatılması, elektron ile safsızlık atomu arasındaki Coulombic etkileşimi artıracak ve bu nedenle safsızlık atomunun bağlanma enerjisi artacaktır. Benzer davranışlar Şekil 3 ve Şekil 4'de görülmektedir.

Olası kızıl ötesi geçişler $\Delta \mathrm{n} \neq 0$ olmak üzere, $\Delta \mathrm{l}= \pm 1$ ve $\Delta \mathrm{ml}=0, \pm 1$ koşulları sonucunda meydana gelir. İlgili iki düzeye ait bağlanma enerjisinin farkının mutlak değerinden elde edilir. Bu koşullara uyan $1 \mathrm{~s}-2 \mathrm{p}_{+}$ve $1 \mathrm{~s}-2 \mathrm{p}$. geçiş enerjileri Şekil 5'de, kuyu merkezinde bulunan donor safsızlık atomu için farklı manyetik alan değerleri ve iki farklı kuyu genişliği $\mathrm{L}=100 \AA \AA$ ile $\mathrm{L}=200 \AA$ için gösterilmiştir. Şekilde görüldüğü gibi manyetik alan geçiş enerjileri üzerinde belirgin bir etki göstermektedir. Manyetik alan yok iken, $\gamma=0$ olduğu için incelediğimiz iki geçiş enerjileri birbirine eşit çıkmaktadır. Ancak kuantum kuyusuna manyetik alan uygulandığında $(\gamma \neq 0), 1 \mathrm{~s}-2 \mathrm{p}_{+}$ve $1 \mathrm{~s}-2 \mathrm{p}-$ geçiş enerjileri farklılık göstermektedir. Manyetik alan etkisi artıkça, $1 \mathrm{~s}-2 \mathrm{p}_{+}$geçiş enerjilerini artarken, $1 \mathrm{~s}-2 \mathrm{p}$ - geçiş enerjileri ise azalmaktadır. Ayrıca büyük kuyu genişliklerinde, geometrik kuşatma zayıfladığı için toplam enerji azalmaktadır ve daha düşük seviyelerde geçiş enerjileri görülmektedir. 
Parabolik kuantum kuyusundaki hidrojenik düzeyler üzerine Manyetik alan etkisi

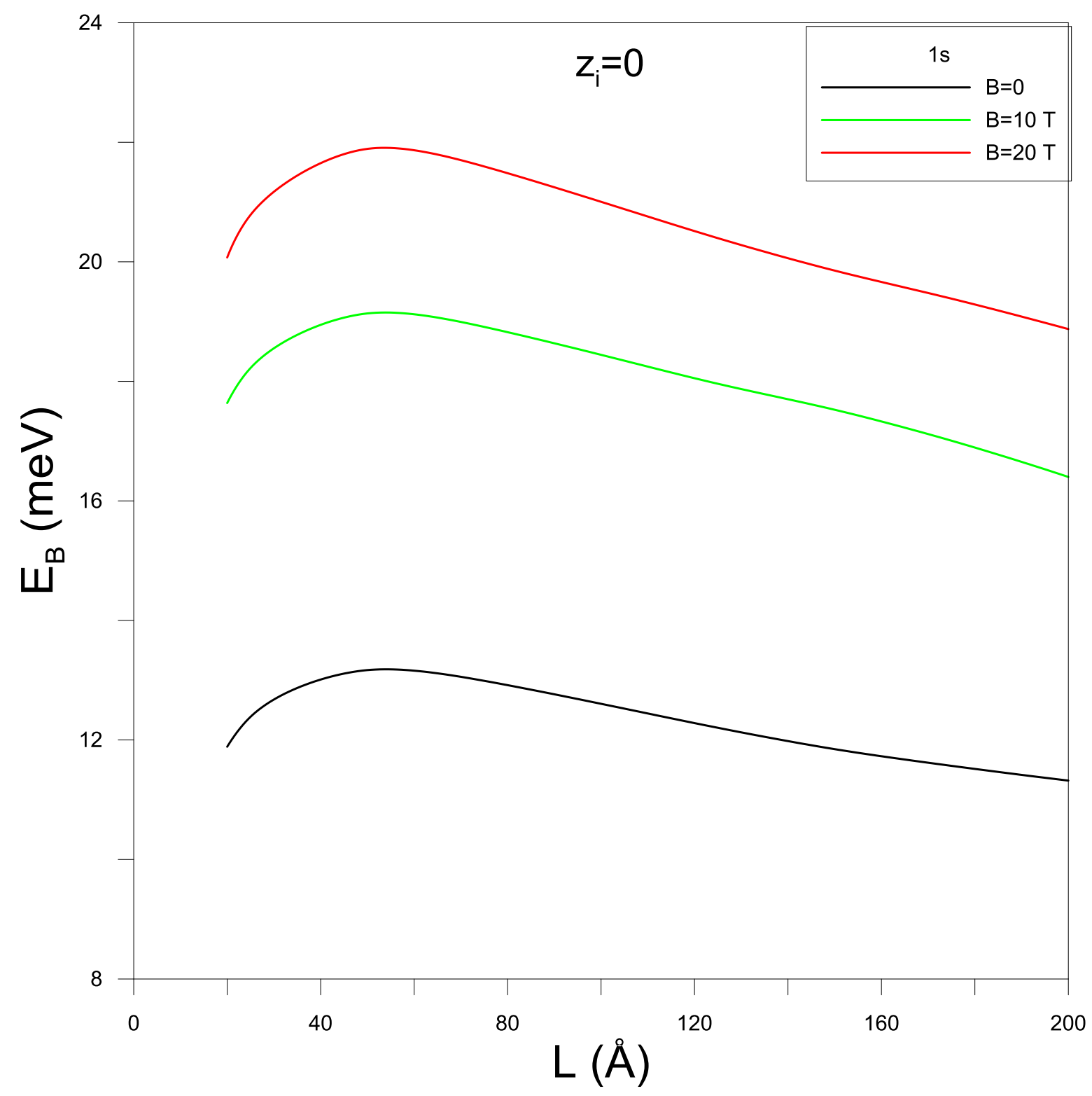

Şekil 2. 1s düzeyindeki bir safsızlık atomunun bağlanma enerjisinin kuyu genişliğine ve uygulanan manyetik alana göre değişimi. 


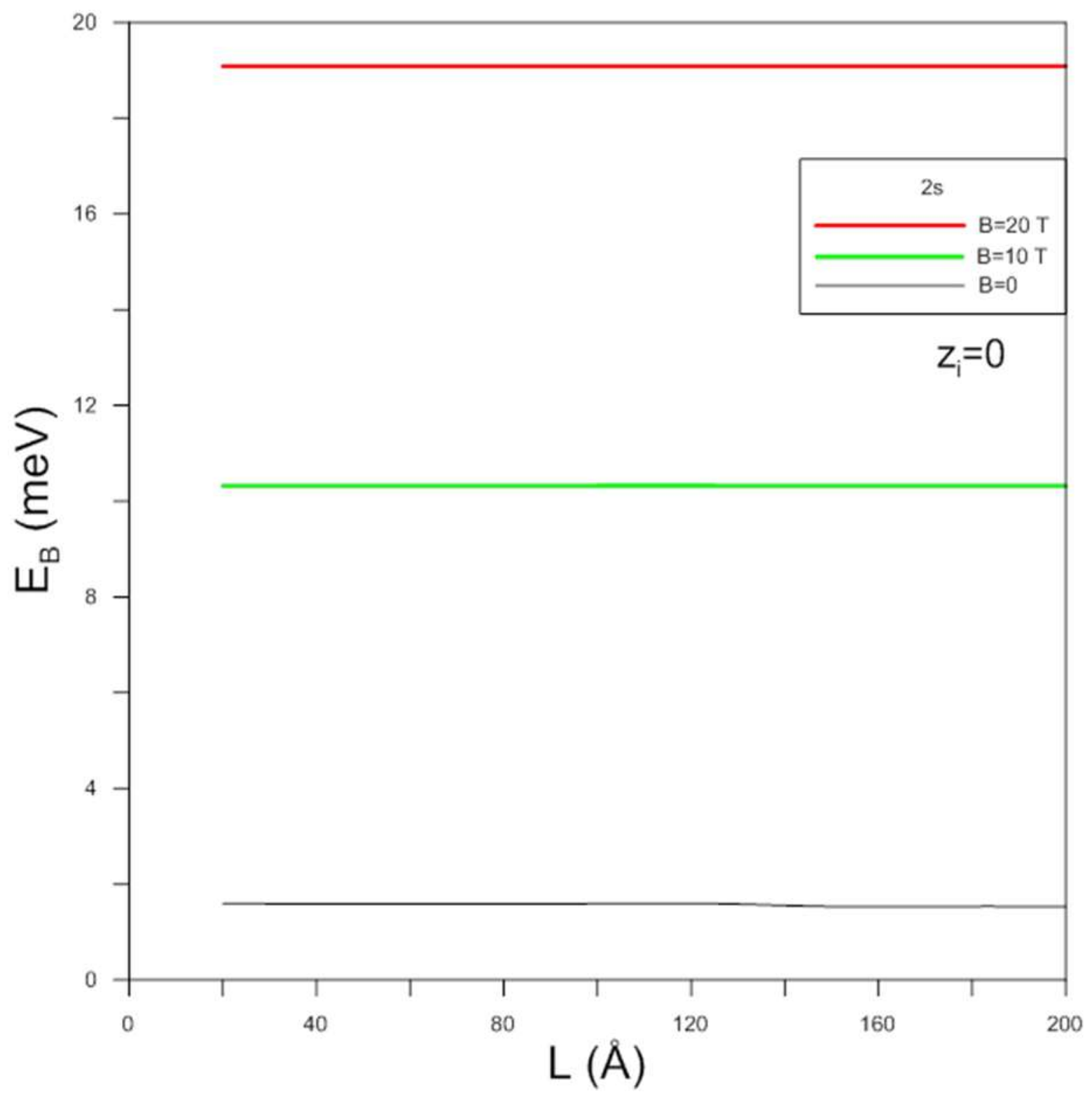

Şekil 3. 2s düzeyindeki bir safsızlık atomunun bağlanma enerjisinin kuyu genişliğine ve uygulanan manyetik alana göre değişimi. 
Parabolik kuantum kuyusundaki hidrojenik düzeyler üzerine Manyetik alan etkisi

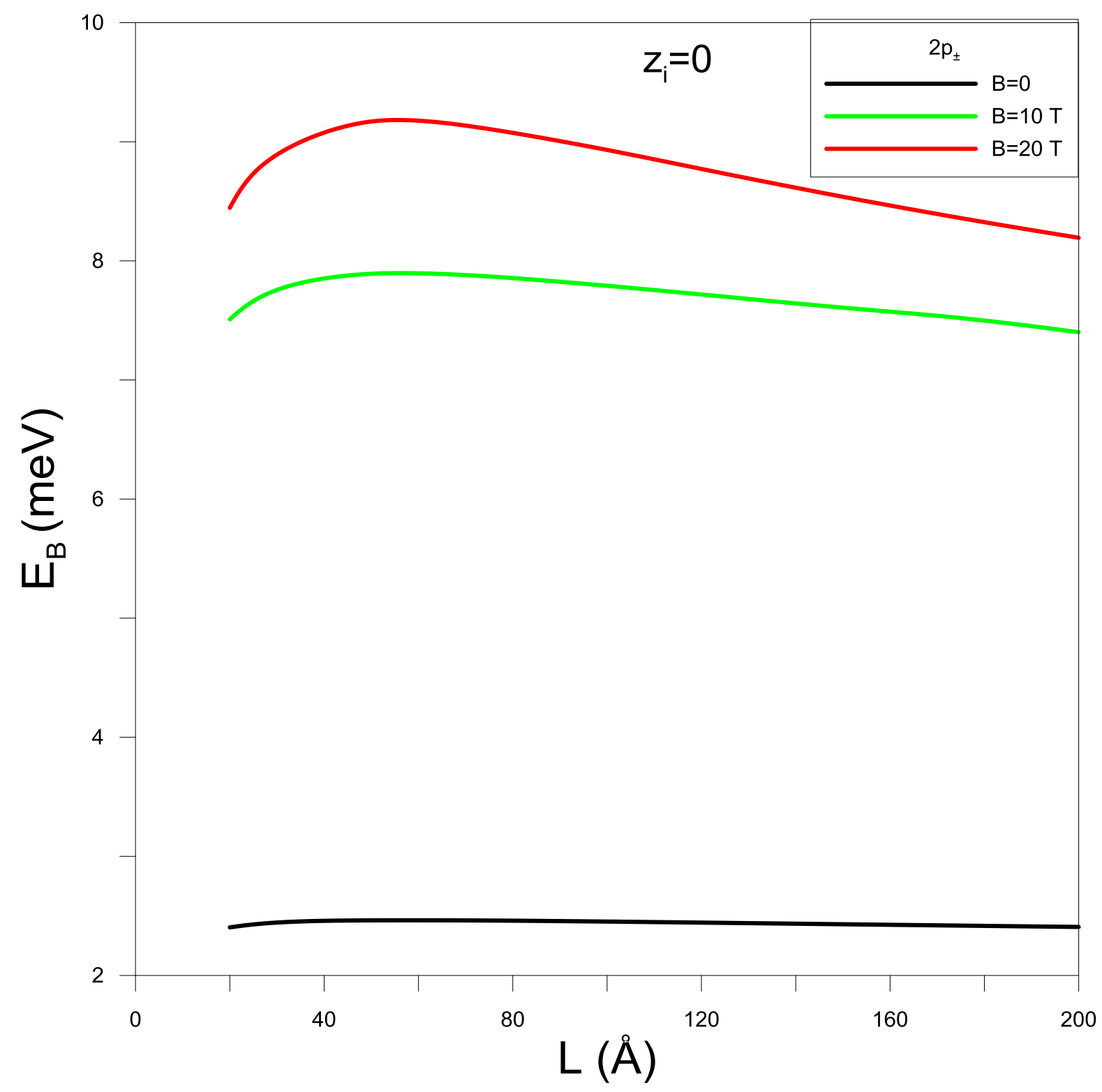

Şekil 4. $2 \mathrm{p} \pm$ düzeyindeki bir safsızlık atomunun bağlanma enerjisinin kuyu genişliğine ve uygulanan manyetik alana göre değişimi. 
YEŞİLGÜL, ÇOBAN, KASAPOĞLU

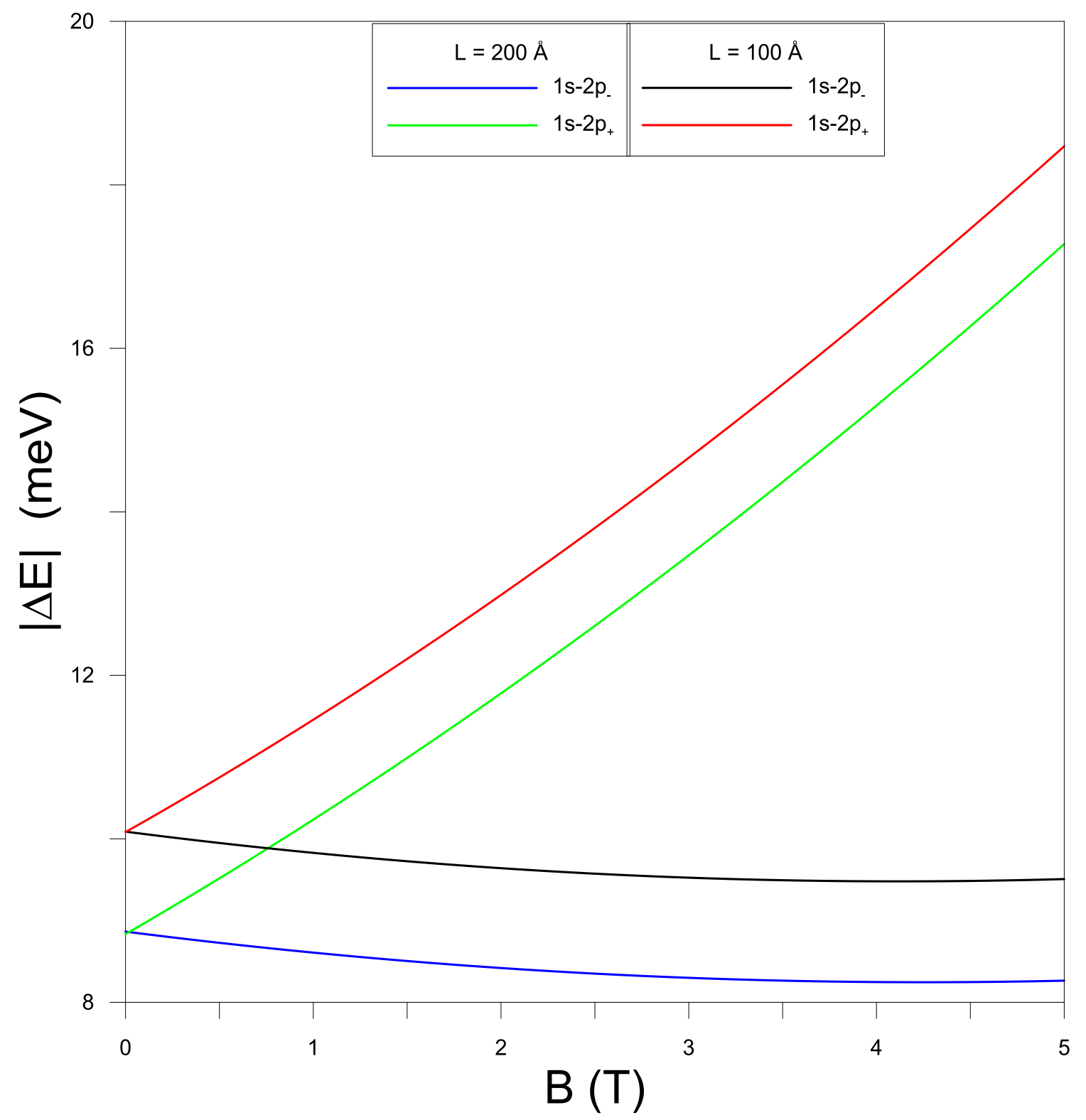

Şekil 5. Sistemin merkezinde yer alan safsızlık atomu için $G a_{1-x} A l_{x} A s / G a A s$ parabolik kuantum kuyusunun farklı kuyu genişliğinin bir fonksiyonu olarak geçiş enerjisinin $(\Delta \mathrm{E})$ manyetik alana göre değişimi.

\section{SONUÇLAR}

Bu çalışmada $G a_{1-x} A l_{x} A s / G a A s$ parabolik kuantum kuyusunda elektronik yapı, hidrojenik donor düzeyleri ve donor düzeyleri arası geçiş enerjileri kuyu genişliği, dışarıdan uygulanan manyetik alanının büyüklüğ̈̈ göre etkin kütle yaklaşımı çerçevesinde varyasyonel hesap tekniği ile incelenmiştir.

Çalışmamızın ilk aşamasında, $G a_{1-x} A l_{x} A s / G a A s$ parabolik kuantum kuyusundaki safsızlık atomunun bağlanma enerjisinin büyütme doğrultusuna paralel yönde uygulanan manyetik alana ve kuyu genişliğine bağlılı̆g incelenmiştir. Büyütme doğrultusuna paralel yönde uygulanan manyetik alanın 
parçacıkların (x-y) düzlemindeki kuşatmasını artırdığı için, bağlanma enerjisinin belirgin bir şekilde manyetik alan ile arttığı gözlenmiştir. Çalışmamızın ikinci aşamasında ise $1 \mathrm{~s}-2 \mathrm{p}_{+}$ve $1 \mathrm{~s}-2 \mathrm{p}$ - düzeyleri arasındaki kızılötesi geçişleri incelenmiştir. Manyetik alanın artması ile $1 \mathrm{~s}-2 \mathrm{p}_{+}$düzeylerindeki geçiş enerjileri artar iken 1s-2p. düzeylerindeki geçiş enerjileri azalma göstermiştir.

Çalışmamızda elde edilen sonuçlara göre ayarlanabilir parametreler değiştirilerek elektronik ve opto-elektronik cihazların geliştirilmesi sağlanabilir. Özellikle ülkemizde de son yıllarda üzerinde yoğun çalışılan güneş pilleri, geniş dalga boyu aralığına sahip veri depolama üniteleri, dedektör, sensör ve diğer terahertz cihazların geliştirilmesinde katkı sağlayacağı düşünülebilir.

\section{KAYNAKLAR}

[1] R.L. Greene, K.K. Bajaj, D.E. Phelps Phys. Rev. B, 29 (1984), p. 1807.

[2] N.E. Kaputkina, Yu.E. Lozovik Physica E, 12 (2002), p. 323.

[3] C.L. Beltran Rios, N. Porras-Montenegro Phys. Rev. B, 68 (2003), p. 155316.

[4] T. Yoshioka, K. Asamo Phys. Rev. B, 86 (2012), p. 115314.

[5] M. Dvorak, S.H. Wei, Z. Wu Phys. Rev. Lett., 110 (2013), p. 016402.

[6] Q. Fanyao, A. L. A. Fonseca, O. A. C. Nunes, J. Appl. Phys. 82 (1997) 1236.

[7] E. Kasapoglu, F. Ungan, H. Sari, I. Sokmen, M.E. Mora-Ramos, C.A. Duque, Seperlatt. Microstruc. 73 (2014) 171.

[8] E. C. Niculescu, L. M. Burileanu, A. Radu, Seperlatt. Microstruc. 44 (2008) 173.

[9] Radu, E. C. Niculescu, M. Cristea, J. Optoelectron. Adv. Mater. 10 (2008) 2555.

[10] L. M. Burileanu, E. C. Niculescu, N. Eseanu, A. Radu, Physica E 41 (2009) 856.

[11] G. Bastard, Phys. Rev. B 24, 4714 (1981).

[12] C. Mailhot, Y.C. Chang, and T.C. McGill, Phys. Rev. B 26, 4449 (1982).

[13] G. Bastard, J. Lum. 30, 488 (1985).

[14] F.I. Betancur, I.D. Mikhailov, Phys. Rev. B 51, 4982 (1995).

[15] E. Kasapoglu, F. Ungan, H. Sari, I. Sokmen, Phys. E 42, 1623(2010).

[16] P. Villamila, C. Cabraa, N. Porras-Montenegro, Microelectronics J. 36,383 (2005).

[17] G.V.B. de Souza, A.Bruno-Alfonso, Phys. E 66, 128(2015).

[18] H. Yıldırım, B. Aslan, Phys. Status Solidi B 249, 2207 (2012).

[19] B. Li, A. P. Djotyan, Y. L. Hao, A. A. Avetisyan, F. M. Peeters, Phys. Rev. B 87, 075313 (2013).

[20] Abramov, World Journal of Condensed Matter Physics 2, 188,(2012).

[21] J. Cen and K. K. Bajaj, Phys. Rev. B 48, 8061 (1993). 